\section{Briginal articleg.}

\section{TIIE CLASSIFICA'TION OF EPILEP'TICS. ${ }^{1}$}

BY W. N. BULLALD, M.1., BOSTON.

Epluepsy is not a disease; it is properly a symptom. Various classifications of epileptics have been proposed, and are used to a certain extent among the writers of treatises on epilepsy and kindred subjects, but none of these has yet become a recognized or standard form. In the very beginning we are confronted by the fact that there are two distinct matters submitted to us, and that the classification of epileptics may be considered from two very different points of view. There is, in the first place, the clinical classification of epileptics - that made according to the type and stage of the disease; the form of classification which should be used in describing or writing about the affection. On the other hand, there is the practical classification of epileptics in institutions, which, so far as it is made at all, has to be made on wholly different grounds.

The clinical classifications of epileptics may be made in various ways, and different elements may be taken as the bases therefor. Before, however, we discuss these we must obtain a clear understanding as to what we mean by epileptics. 'Tho word "epilepsy" may be used in a wide or in a narrow sense. In the one case it is synonymous with the so-called essential or idiopathic epilepsy. In the broader sense it is used to comprehend essentially all cases in which epileptic or epileptiform attacks occur more than once, or more than a few times. Some writers exclude from the term "epilepsy" certain conditions, as those, for example, in which well known organic lesions of other portions of the body than the intracranial contents form the starting point or basis of the affection, and as some authors include more and others fewer of these conditions, the word "epilepsy" in its broader sense has at the present time ill-rlefined limits. For our purposes, however, it will be best to include under this signification all cases of persons suffering from epileptic or epileptiform convulsions, in which these are to be considered either as illiopathic or as due to primary intracranial conditions. By this definition we exclude epileptiform convulsions directly due to cardiac or renal disease.

The usual methods of classifying epileptics clinically are (1) according to etiology ; (2) according to symptoms.

The etiological classification is undonbtedly the most scientific, and is, on the whole, the best form of what I have termed "clinical classification." The principal objection to this method is its lack of certainty. In the first place, there are many cases in which, although a definite cause can be detected, either post mortem or at some time during the life of the patient, nevertheless this cannot be detected or learnt at the time when the patient is under observation. Moreover, there are many cases in which the existence of the epilepsy is falsely attributed by the patient or his relatives to a supposed cause. As in almost all cases, some of the tramatic excepted, we have no physical signs on which to rely we are obliged to depend on the history of the case and are largely at the mercy of the narrators. Again, there is a large class, the so-

1 Read beforo tho Now Eugland Paychological Society, March 10, called idiopathic epilepsy, in which even with our best methods in post-mortem examination we can find no organic lesion which will account for the symptoms. For these reusons, the etiological classification is in the present state of our knowleige often diflicult, occasionally impossible. While fully recognizing that epilepsy itself is a symptom of some abnormal intracranial condition, we are frequently at a total loss to know $(a)$ what that condition pathologically is; (b) what has produced that pathological condition. Macroscopically, at the autopsy, we can discover nothing, and even microscopical examination of the brain tissues gives us no satisfactory information.

In spite, however, of these objections, which are practically set aside as far as possible, by placing all cases in which no definite etiology appears in a class by themselves, this is the most convenient and the most scientific method of classification for clinical purposes. In this system we essentially divide all epileptics into the two classes of (1) idiopathic or true epilepsy, where no definite cause for the affection can bo detected; (2) all other forms of epilepsy for which definite causes exist. Class (2) contains a number of definite divisions :

(A) Traumatic, where the epilepsy is directly due to injury. In this division should bo included not only the cases $(a)$ in which the brain or its members are torn or otherwise injured, or where the epilepsy is the result of an intracranial hemorrhage, but also (b) those in which there is a continued increased pressure on the brain and meninges, as by depressed bone, and lastly $(c)$ those more obscure cases, in which epilepsy directly follows a blow on the head, although no organic lesion can be detected either during or after life. In some of these latter cases it is probable that the signs of the injury have disappeared; in others it is possible that no visible permanent sigus existed; in many we have no opportunity for intracranial investigation.

(B) The second subdivision of epilepsy is the toxic. The common type of this form is the so-called alcoholic. It is generally admitted that a form of epilepsy exists which is primarily due to the abuse of alcohol. I must confess that I have never myself scen a case in which, after due consideration, I am now convinced that this cause alone produced epilepsy, but it must be mentioned here, as the vast majority of writers acknowledge its existence. On the other hand, there is no doubt that epileptic convulsions are caused by absinthe, the essential oil being here the prominent factor. In certain other cordials, also, the essential oil causes the same results.

Lead may also be a cause of epileptic couvulsions, but it is a rare one, and usually as a result of definite lesions. Convulsions from lead indicate a very severe poisoning and suggest a fatal termination.

$(C)$ Organic epilepsy is frequently the result of non-traumatic lesions of the intracranial contents. These lesions may be due to more or less localized causes, as, for example, to hemorrhage from some of the intracranial vessels, or to embolism, or thrombosis in one or more of them. It may bo and not rarely is caused by neoplasm in the bruin or meninges, aind it not infrequently, especially in children, is found in connection with cerebral sclerosis. Its occurrence in paralytic dementia, another form of sclerosin, is too well known for me to do more than to mention it here, and state that in this affection it may be due to 
various lesions, the results of endarteritis, cerebral hemorrhages, or possibly the sclerosis itself.

Another form of organic epilepsy is seen in that occurring in tubercular tneningitis, where the convulsions may be due apparently to more or less diffuse conditions (meningitis) or to localized ones (tumor, collections of miliary tubercles in brain or meninges).

$(D)$ Perhaps the most important form of epilepsy of which we know the cause is the syphilitic. In this we can often find at autopsy some definite lesion which may be looked upon as the cause. 'The most common is the syphilitic endarteritis with its results; much more rarely it occurs from gummata; it is not common from uncomplicated meningitis. On the other hand, there are undoubtedly a certain number of cases of epilepsy due to syphilis in which lesions are not found post mortem. How large this number is we can only surmise. Clinically many cases of epilepsy are presented to us in which the question of syphilitic origin has arisen, but cannot be determined. In cases due to tumor, in cases where the attacks have come on suddenly and in considerable numbers, and where other syphilitic manifestations or a syphilitic history is present, we can often readily make the diagnosis, but where these conditions are not found, where a single attack occurs without known cause, unaccompanied by any other special symptoms and not followed by other attacks perhaps for months, the diagnosis is often extremely difficult, very often impossible. Specilic treatment is sometimes, but not often, of value.

These are the principal classes of epilepsy divided according to etiology. (Other forms are recognized, which, however, can easily be arranged in the proper category. In some cases we have apparently rather an exciting than the determining cause and we must exercise care in distinguishing between these. In persons who for any reason are predisposed to epilepsy au uttack may be induced by exciting causes which would not suffice to produce iny objective result in a perfectly sound body. Such probably are many at least of the cases called reflex, in which the apparent existing cause is a continued irritation of some peripheral nerve or nerves. I recollect well that BrownSequard in one of his lectures in Boston referred to a case which was, in his opinion, caused by some chronic irritation of one toe, -1 believe an in-growing toe nail.

Another form of clinical classification of epileptics is the symptomatic. 'This is exceedingly simple, and is much used as being the easiest and realiest method. It has, however, two objections. One is a very serious one. It is that it is totally unscientific, and so far as it implies, in the case of certuin symptoms, any essential difference in the nature of the affection would seem to be wholly fallacious. The nature of the affection remains the same, whether it manifests itself for the time being as pet $t$ mal, as grand mal, or as a condition of automatic action. (On the other hand, there are certain symptoms about the nature of whose cause we are in doubt, which may be due to the same condition which forms the basis for the grand mal or petit mal, or may be due to other conditions. Some of these symptoms or symptom-complexes may be independent, that is, they may occur by themselves without connection, so far as can bo seen, with any other symptomcomplexes, and the question then naturally arises as to whether they are metabolic or substitutive forms of epilepsy. Others occur not alone in connection with epilepsy, but are also concomitant with other affections, as chorea, insanity or allied troubles. The second objection to this form of classification is that it has not yet been decided by general medical opinion whether certain symptoms and symptom-complexes should be classed under epilepsy or not. 'This is at present done, or not done, according to the personal views of the observer, and sometimes may cause difficulty in the comparison of statistics.

The essence of the classification of epileptics by symptoms (symptom-complexes) consists in taking certain prominent symptoms as the bases and in placing all those patients in whom one of these symptoms predominates in its respective class.

'The three primary classes in which epileptics should be arranged according to this classification are (1) that in which graud mal is the prominent feature; (2) that in which petit mal is the prominent feature; (3) that in which automatic actions are the prominent feature. Each of these classes may have numerous subdivisions according to the pleasure of the observer. Thus we may divide the cases of grand mal into those in which an aura exists, and those in which it is not found. We may divide the automatic actions according to their character, as rumning, walking, or more elaborate and complicated actions.

As said before, however, all these subdivisions are futile, spurious and artificial. They should be abolished, and this form of classification ought to fall into disuse.

The practical classification of epileptics in an institution established for them rests, however, upon entirely different principles. The arrangement to be adopted in uny given institution will naturally depend to a considerable extent upon the size and number of buildings to be occupied by patients, and upon the size, number and position of the various dormitories and rooms. For our present purpose we will imagine a theoreticnlly perfect institution, and assume that any desirable arrangements can be made. In all institutions there are divisions which must always be made. These are determined by practical considerations. First and most obvious is the division by sexmales and females. Next to this, as we assume our institution to take all classes of epileptics, must a division be made into the sane and the insane. There will always be a considerable number of patients who will stand clgse to the border line of these divisions, and there will be in any large institution of this character many whom we shall feel it best to move from time to time from one of these divisions to the other. Thus not only will the exact limit of these divisions from the point of view of the superintendent be somewhat indefinite, but also the numbers in each division with only the same patients in the institution will be constantly fluctuating. Considering now only the insane epileptics we shall find that they must be divided roughly into several groups: (1) the criminal; (2) the habitually violent; (3) the temporarily violent; this third class includes those persons who are habitually either mildly insane, or in such condition as not to be dangerous to themselves or others, but who are in the habit of having violent outbreaks for a comparatively short time, from which they return to their usual condition; (4) the mild, or non-violent, non-criminal ones.

Among the mild insane epileptics another division may be made according to the degree of insanity. We may roughly divide them into three classes: (1) the 
much demented and helpless; (2) the moderately demented, harmless epileptics, able to get about somewhat, and perhaps to do light work; (3) those who are but slightly demented or essentially sane between the attacks, who, as a class, can do moderately heavy or regular heavy work; if men, heavy laboring work.

Wo have also the sane epileptics, who are to be considered in this connection, many of whom can not only do ordinary heavy laboring work, but among whom we shall find skilled mechanies, and those who have learned and practised various trades. These persons are still able to practise, of ten with good success, their former occupations, if only the opportunity be given them.

Epileptics in an institution must also be classified to a certain extent clinically, according to the number and character of their attacks. Patients having frequent attacks of petit mal often need considerable supervision, and cannot be entrusted with dangerous machinery or tools. Those much affected with grand mal are even more to be guarded, and cannot be allowed alone without aid in any place where a sudden fall would be dangerous.

'There are certain executive considerations in arranging for epileptics in any institution which receives all persons suffering with this affection. One which is of practical importance, and which applies to both the Banc and insane, is the question of expense. Our theoretical institution must receive not only those patients who are supported at the expense of the State, county or town, but also private patients who may pay any price "up to fitiy dollars a week.

$\Lambda$ s many of our patients are supposed to be capable of work, they may be classified for statistical purposes in regard to their abilities in this division. 'This may be an important consideration in regard to their location and arrangement in the institution, especially when taken in connection with other factors.

I offer these considerations, gentlemen, as a basis for discussion and information in regard to the best practical divisions for epileptics in institutions.

\section{JUSTUS'S 'TEST FOR THE MIAMNOSIS OF SYIHILIS.}

BY monAlen e. ramot, M.D., mostun,

Ihysician to Out-Patients at the Massachusetts Gincral Hospital,

ANI P. H. Min'rins, M.D.,

(), the Harvard Nedical Sehool

Is two papers " published in 1895 and 1897 respectively a new test for the diagnosis of doubtful cases of sy philis was described by Justus, an assistant in Schwimmer's clinic at Budapest. 'The test depends upon the sensitiveness of the red blood corpuscles in syphilitics to the action of mercury exhibited by inunction, or by subcutaneous or intravenous injection. This sensitiveness, which is suid by Justus to be greater in syphilis than in any other disease, is shown by a sudden sharp fall in the percentage of hemoglobin during the hours immediately following the administration of the drug. Later the hemoglobin gradually rises to a point above that seen before the injection. Such a sharp fall of 10 to 20 per cent. in the hemoglobin following the use of mercury was observed by Justus in over 300 cases of syphilis, and the absence of any such fall in non1 Juslus : Virchow's Arohtr, vol. 140, y. 01, 1895; also vol. 148, p.
633, 1897. syphilitic cases was attested by "very numerous" con. trol experiments.

The sign was observed in all untreated cases of the secondary, tertiary or congenital form of the disease, and in 13 out of 16 cases in which only a fresh chancre and inguinal adenitis were present. "I atent cases," so called, showing no lesions, and cases in which the lesions have passed their acme and were subsiding, either spontaneously or under treatment, do not react

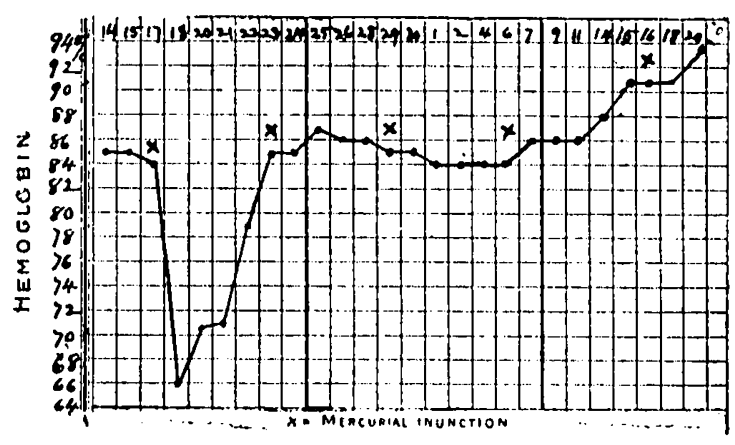

Cha rét.

characteristically to the test. The three cases of fresh chancres above mentioned were in the stage of subsidence - nearly healed. It is therefore in florid cases with alvancing lesions of whatever type that the test is positive. 'This includes relapses of all kinds so long as the lesions are in an active condition.

The amount of the fall in hemoglobin after a given dose of mercury depends, according to Justus, on the severity of the disease and the condition of the patient.

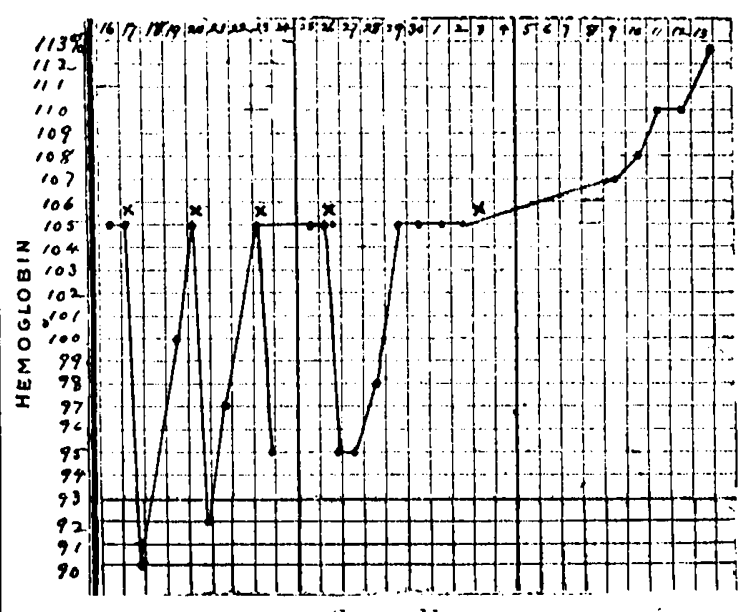

CHAкT 11.

The same conditions determine how soon the blood shall return to its formal condition.

If a subcutaneous or intravenous injection is given the characteristic sharp fall in hemoglobin usually follows each of the first three or four doses (see Chart I), while if the method of inunction is used the hemoglobin falls only after the first inunction. If the drug is given by mouth no effect on the blood is to be observed. Intruvenous injections act so rapidly that within an hour a loss of 15 per cent. of coloring matter has been noted. Microscopic examination of the 